\title{
Convergencias entre la rentabilidad y la liquidez en el sector del agronegocio*
}

\author{
Convergences between profitability and liquidity in the agribusiness sector \\ Convergências entre rentabilidade e liquidez no setor do agronegócio
}

Xiomara Esther Vazquez Carrazana a

DOI: https://doi.org/10.11144/Javeriana.cc18-45.crls

Universidad Federal de Uberlândia, Brasil

xesther68@gmail.com

Redalyc: http://www.redalyc.org/autor.oa?

$\mathrm{id}=0000-0002-2338-337 \mathrm{X}$

Fecha de recepción: 13 Abril 2017

Ilirio José Rech

Universidad Federal de Uberlândia, Brasil

ORCID: http://orcid.org/0000-0001-7027-737X

Gilberto José Miranda

Universidad Federal de Uberlândia, Brasil

ORCID: http://orcid.org/0000-0002-1543-611X

\author{
Marcelo Tavares \\ Universidad Federal de Uberlandia, Brasil \\ ORCID: http://orcid.org/0000-0003-3008-3460
}

Fecha de aprobación: 18 Mayo 2017

\section{Resumen:}

En muchos casos, los indicadores de rentabilidad y liquidez han sido evaluados de acuerdo con una relación de rivalidad. Sin embargo, un análisis concienzudo de la interacción de estas variables sugiere la coexistencia y no la contraposición -término de por sí problemático y que será sometido a evaluación en el presente artículo- de ambas. Este artículo tiene el objetivo de evaluar la relación que se entabla entre la rentabilidad y la liquidez en empresas de agronegocio. El análisis desarrollado tiene las características de un estudio descriptivo cuantitativo; cuenta con el empleo de métodos de análisis de correlación y segregación; corresponde a una muestra de 20 empresas del sector del agronegocio brasileño listadas en la base de datos Economática, y atañe al período comprendido entre 2005 y 2015 . El estudio realizado permitió observar que la correlación que existe entre la rentabilidad y la liquidez, en el marco definido, fue positiva y significativa. Por su parte, los resultados de la prueba Mann-Whitney sugieren que la composición y la rotación de los activos fijos constituyen variables que diferencian el comportamiento de las empresas de este tipo. En suma, pueden ser factores determinantes al momento de direccionar la coexistencia de la rentabilidad y la liquidez en el sector del agronegocio. Palabras clave Rentabilidad y liquidez; rentabilidad del agronegocio; composición de activos en agronegocio.

Código JEL M41

Palabras clave: Rentabilidad y liquidez, rentabilidad del agronegocio, composición de activos en agronegocio.

\section{Abstract:}

In many cases, the profitability and liquidity indicators have been evaluated as per a relationship of rivalry. However, a thorough analysis of the interaction of these variables suggests for both coexistence and not opposition -which, in itself, is problematic term that will be subject to evaluation in the present article-. This article aims to assess the relationship between profitability and liquidity in agribusiness companies. The analysis developed shows characteristics of a quantitative descriptive study; makes use of analysis of correlation and segregation methods; corresponds to a sample of 20 companies from the Brazilian agribusiness sector listed in the Economática database; and pertains to the period between 2005 and 2015. This study made possible to observe that the correlation between profitability and liquidity, within the defined framework, was positive and significant. On the other hand, the results of the Mann-Whitney test suggest that the composition and rotation of fixed assets are variables that differentiate the behavior of companies of this type. In short, they can be determining factors when addressing the coexistence of profitability and liquidity in the agribusiness sector.

Notas de autor 
Keywords: Profitability and liquidity, profitability of agribusiness, composition of assets in agribusiness.

Resumo:

Em muito caso, os indicadores de rentabilidade e liquidez foram avaliados de acordo com uma relação de rivalidade. No entanto, uma análise afincada da interação dessas variáveis sugere a coexistência e não a contraposição -termo de por si problemático e que estará sujeito a avaliação no presente artigo- de ambas. Este artigo objetiva avaliar a relação estabelecida entre a rentabilidade e a liquidez em empresas de agronegócio. A análise desenvolvida possui as características de um estudo descritivo quantitativo; tem o uso de métodos de análise de correlação e segregação; corresponde a uma amostra de 20 empresas do sector do agronegócio brasileiro listadas na base de dados Economática, e atinge ao período compreendido entre 2005 e 2015 . O estudo realizado permitiu observar que a correlação existente entre rentabilidade e liquidez, no marco definido, foi positiva e significativa. Por sua vez, os resultados do teste de Mann-Whitney sugerem que a composição e rotação de ativos fixos constituem variáveis que diferenciam o comportamento das empresas deste tipo. Em suma, podem ser fatores determinantes no momento de direcionar a coexistência da rentabilidade e a liquidez no setor do agronegócio.

Palavras-chave: Rentabilidade e liquidez, rentabilidade do agronegócio, composição de ativos em agronegócio.

\section{Introducción}

El punto de partida del presente análisis son los abundantes estudios teóricos y prácticos que se han dedicado a las contraposiciones que se establecen entre la liquidez y la rentabilidad. Dichos estudios conforman antecedentes importantes que llevan a pensar que la controversia que se cierne entre estas dos variables podría encontrar salida si se estudiaran los niveles idóneos de elementos comunes a ellas. En ese sentido, cabe observar de entrada que la mencionada perspectiva de contraposición se fundamenta en el enfoque tradicional de las finanzas, el cual se orienta hacia el imperativo de mantener una relación positiva y con carácter creciente de capital de trabajo; enfoque al que se opone una perspectiva según la cual demasiada liquidez acarrea menor rentabilidad (Sánchez \& Bernabé, 2002; Deloof, 2003; Padachi, 2006; Lazaridis \& Trifonidis, 2006).

A pesar de que los niveles adecuados de estos dos indicadores sean elementos fundamentales para la supervivencia y desarrollo de las organizaciones económicas, la relación mostrada entre ellos es tradicionalmente negativa (Jarvis, Kitching, Curran \& Lightfoot, 1996; Eljelly, 2004). Asimismo, la contraposición entre liquidez y rentabilidad es reconocida como el dilema fundamental de la contabilidad (Pimentel, Braga \& Pereira, 2005).

En tanto, la relación negativa que se cierne entre la liquidez y la rentabilidad encuentra explicación en que el crecimiento de activos circulantes es directamente proporcional al de la liquidez: se trata de activos que están asociados negativamente al retorno de la inversión, no solo por encontrarse en el denominador de la forma de cálculo, sino porque precisamente por ser activos circulantes tienen menores capacidades para generar utilidades (Hirshleifer, How, Teoh \& Zhang, 2004). Cabe anotar que un exceso de activo circulante merma la rentabilidad de la inversión por dos vías complementarias. Varios autores coinciden en que, como consecuencia de dicho exceso, por una parte, se contemplan mayores costos operativos y, por otra, se maneja un mayor volumen de inversión (Braga, Nossa \& Marques, 2004; García \& Martínez, 2007; Rahneman \& Nasir, 2007; Samiloglu \& Demirgünes, 2008; Pérez, Castro \& García, 2009; Talha, Christopher y Kamalavalli, 2010). En general, los resultados de diferentes investigaciones sugieren que el efecto del retorno de activos se relaciona con la política asumida para la gestión del capital de trabajo (Jarvis et al., 1996; Talha et al., 2010; Dong \& Su, 2010; Ikram, Mohammad, Khalid \& Zaheer, 2011; Hussain, Farooz \& Khan, 2012; Mehtab \& Marsap, 2015; Anake, Ikes \& Menyo, 2015). La literatura ha centrado su atención en los componentes del capital de trabajo para explicar cambios en la rentabilidad, de tal modo que el estudio de la influencia de márgenes y rotaciones queda relegado a un segundo plano (Monterrey \& Sánchez, 2011). Asimismo, en la literatura también es posible encontrar evidencias que apuntan hacia una relación positiva entre los dos indicadores centrales en este estudio. 
Los estudios correspondientes a las correlaciones positivas entre la liquidez y la rentabilidad muestran resultados diversos. En primer lugar, cabe destacar el caso de las empresas familiares de la asociación portuguesa, que poseen mayores posibilidades de enfrentar sus obligaciones de pago cuando presentan mayores niveles de rentabilidad, dado que están orientadas hacia una política económica según la cual las relaciones entre estos dos conceptos deben ser positivas (Correia, 2003). Más adelante, en el estudio de Melgarejo y Vera (2010) se encuentra una relación positiva entre la rentabilidad y la liquidez en sociedades laborales y mercantiles convencionales de Navarra, España. Asimismo, Pimentel y Lima (2011) encontraron relaciones temporales positivas entre ambas variables en el sector textil de Brasil. En ese contexto, cabe añadir que los términos contraposición o rivalidad no son los que mejor expresan una comprensión de la relación que hay entre la rentabilidad y la liquidez. Por una parte, la rentabilidad no ofrece resistencia a la liquidez (Aguilar, Ruíz y Santana, 2000; Pimentel y Lima, 2011). Por otra, un estudio de las múltiples relaciones que se entretejen con la rentabilidad, permitirá encontrar explicaciones para los niveles requeridos de liquidez (Koshio \& Nakamura, 2013).

Una vez establecido el contexto de acuerdo con las ópticas de los aportes aludidos, puede añadirse que el objetivo de este estudio es evaluar la relación existente entre la rentabilidad y la liquidez en empresas pertenecientes al sector del agronegocio. Con ese objetivo, una muestra de 20 empresas brasileñas listadas en la base de datos Economática y el resultado de los indicadores en estudio en el período comprendido entre los años 2005 y 2015 , fueron analizados.

Como se expondrá, este estudio evidencia la posibilidad de una correlación positiva entre las variables de rentabilidad y liquidez en las empresas del sector del agronegocio brasileño. Lo anterior confirma la posibilidad de que la flexibilidad que hay entre estos dos indicadores sea posible (Iudícibus, 1998), y de que la composición de activos sea una variable a considerar para la evaluación de la tenencia de una posición económico-financiera equilibrada (Padachi, 2006; Padoveze \& Benedicto, 2007; García \& Martínez, 2007; Rahneman y Nasir, 2007; Afza \& Nazir, 2007; Samiloglu \& Demirgünes, 2008; Mathuva, 2009; Porlles, 2015).

\section{Liquidez y rentabilidad}

Con el fin de analizar la interacción que tienen las variables centrales del presente estudio, cabe delinear definiciones de ambas. Así pues, la liquidez es entendida como la flexibilidad en el cambio de activos por otros más líquidos, en un contexto en el que esta asegura el pago de las obligaciones de la empresa (Vercelli, 2000; Souza, 2008): en consecuencia, se perfila como una condición para la continuidad del ciclo efectivo-inversiónefectivo (Cuervo \& Rivero, 1999; Hasbrouck y Seppi, 2001; Pimentel, Braga \& Pereira, 2005; Matarazzo, 2008; Fernández, Ferreira \& Rodríguez, 2014). Por su parte, los niveles de rentabilidad que obtiene una empresa revelan la eficacia de la administración para generar utilidades a partir de la inversión en activos, de tal modo que mayores niveles de esta variable evidencian las cualidades de la compañía para ofrecer un retorno del capital empleado (Jiménez y Lozano, 2014). En consecuencia, este indicador es usualmente considerado la medida de mayor relevancia para juzgar el acierto o fracaso de una empresa determinada (Acosta, Correa \& González, 2002; Pimentel, Braga \& Pereira, 2005).

En suma, los resultados de ambos indicadores configuran la salud empresarial de una compañía (Iudícibus, 1998), en un contexto en el que para las empresas no siempre resulta posible enfrentar sus obligaciones de pago y ofrecer un retorno adecuado de sus activos (Amat, 2001; Hasbrouck y Seppi, 2001; Chordia, Roll, \& Subrahmanyam, 2000; Gill, Biger \& Mathur, 2010). 


\section{Interacciones entre la liquidez y la rentabilidad}

Altos niveles de rentabilidad refuerzan la liquidez. La tenencia de mayor efectivo no solo es consecuencia de una mayor rentabilidad sino que también es su causa (Pimentel, 2008; Koshio \& Nakamura, 2013). En ese sentido, la rentabilidad posee la virtud de envolver todos los elementos operacionales en un modelo único, lo cual abre las posibilidades para encontrar factores afines a la liquidez (Marques \& Braga, 1995; Braga et al., 2004; Padoveze \& Benedicto, 2007; García \& Martínez, 2007).

En consonancia, la rotación de activos constituye un factor esencial en nuestro estudio, dado que influye sobre la rentabilidad de forma directa y, simultáneamente, posee elementos que explican la liquidez (Martins, Miranda \& Alves, 2014). Asimismo, en el marco de la discusión que se entabla entre el riesgo y la rentabilidad, fue defendida la existencia de una relación de causalidad entre ventas y activos circulantes. La demanda de ventas es la causa de que se invierta en inventarios, cuentas por cobrar y en la mantención de efectivo (Shin \& Soenen, 1998; Howorth \& Westhead, 2003; Medeiros \& Rodrigues, 2004; Deloof, 2003). Ante ese panorama, cabe formular que las variaciones del volumen de ventas transforman el activo circulante, en tanto que mayores niveles de activos circulantes pueden estar relacionados con mayores niveles de ventas.

El nuevo ingrediente que corresponde a las ventas le otorga una mayor complejidad a la aproximación hasta tanto desarrollada. Si bien este indicador influye de forma positiva sobre la rentabilidad, los activos circulantes surten efectos paralelos en el sentido inverso: una aparente paradoja que se resuelve en la medida que se encuentren las proporciones adecuadas que conectan a los activos circulantes y los activos fijos (Filbeck $\&$ Krueger, 2007). En ese contexto, disminuir el nivel de inversión en activos circulantes cuando sea posible sostener las ventas como consecuencia de mayores rotaciones de los activos fijos, puede conducir a una relación de convergencia entre liquidez y rentabilidad (Mathuva, 2009; Mohammadi, 2009; Mona, 2012; Van Horne \& Wachowicz, 2014; Muhammad \& Akin, 2015; Porlles, 2015). La observancia de este panorama, con sus múltiples componentes y matices, muestra la verdaderas dimensiones del análisis que se busca desarrollar.

\section{Estructuras de activos en el agronegocio}

Una de las particularidades de las empresas de agronegocio corresponde a su conceptualización. En su conjunto, estas se perfilan como aquel grupo de empresas cuya actividad consiste en la combinación de recursos humanos, financieros y naturales que tiene el objetivo de transformar recursos biológicos para obtener productos agropecuarios o activos biológicos adicionales. De ese modo se distingue la presencia de los llamados activos biológicos, que se caracterizan por aumentar el rubro del balance financiero, y cuya presencia repercute en el resto de los indicadores económico-financieros (Barry, Ellinger, \& Hopkin, 1995; Hall, Beck $\&$ Toledo, 2012).

La influencia de los componentes del activo circulante y de los factores productivos de este tipo de actividad, es diferente de la observada en otras. En la actividad agrícola los tiempos de crecimiento, desarrollo y obtención del producto prácticamente constituyen condicionantes que atañen a la inmovilización de recursos invertidos, debido a los inexorables pasos de la naturaleza y los tiempos del propio proceso productivo (Symons, 2005; Brito, 2010).

En ese contexto, el agronegocio involucra a un conjunto de agentes vinculados con la producción, la distribución, la comercialización y el procesamiento de productos agropecuarios (Cáceres, 2015). Los nuevos enfoques tecnológicos en este sector condicionan el empleo de una estructura de capital diferente (Caligaris, 2015). Simultáneamente, la producción agraria se caracteriza por desarrollarse bajo una serie de condicionamientos naturales específicos que afectan la valorización normal del capital: la propia dinámica de funcionamiento ha conllevado a que este tipo de empresas tengan una mayor relación de capital circulante/ 
fijo, mientras que el carácter particularmente prolongado del proceso de producción agrario afecta el tiempo de rotación y la movilidad del capital (Vértiz, 2015).

De acuerdo con circunstancias como las anteriormente descritas, muchas empresas acuden a la contratación de las tareas vinculadas a la siembra, las labores culturales, la cosecha y el transporte de las mercancías agraria: una forma de apropiación de los elementos productivos que torna al conjunto del capital adelantado en un capital con elevados valores de activos circulantes (Caligaris, 2015).

Cabe mencionar que las empresas agropecuarias requieren contratar menos empleados permanentes y a menudo tercerizan las actividades productivas con compañías prestadoras de servicios especializados. Esta nueva estructura gerencial tiene un impacto significativo en la forma como se compone el capital de las empresas agropecuarias, y acarrea una profunda transformación en el sector agropecuario, que trasciende el campo tecnológico y redefine cualquier tipo de análisis (Cáceres, 2015).

\section{Metodología}

\section{Definición de objetivo y tipo de estudio}

La posibilidad de que una determinada composición de activos pudiera constituir una de las condiciones esenciales para la convergencia de la rentabilidad y la liquidez, fue motivación importante para la presente propuesta de evaluación de la relación existente entre ambas variables en empresas pertenecientes al sector de los agronegocios. Dado que las empresas de este tipo tienen niveles de activos circulantes altos, tanto por los cambios ocurridos en la generación del capital como por su composición, que está basada en activos biológicos corrientes y en inventarios con una lenta rotación, el presente estudio parte de la consideración de que la rotación de los activos fijos en estudio propiciará ritmos tan acelerados como para poder cubrir la necesaria e ineludible tenencia de los activos circulantes.

El estudio desarrollado tiene las características de un análisis descriptivo cuantitativo que se sirve de métodos de análisis de correlación y segregación del período comprendido entre 2005 y 2015. Como se mencionó con anterioridad, en él se utiliza una muestra de 20 empresas pertenecientes al sector del agronegocio de Brasil, y se realizan 231 observaciones en total.

\section{Variables empleadas y forma de cálculo}

Las variables empleadas y su forma de cálculo pueden ser observadas en el tabla 1. 
TABLA 1

Variables empeladas y formas de cálculo (\$)

\begin{tabular}{l|l}
\hline Identificación & Fórmula \\
\hline AO (Activo operativo) & Activo fijo + Capital de trabajo \\
\hline RAO (Retorno de activo operativo) & Utilidad operativa / Activo operativo \\
\hline RA (Rotación de activo ) & Ventas / Activo operativo \\
\hline MO (Margen operacional) & Utilidad operativa / Ventas \\
\hline RAF (Rotación de activo fijo) & Ventas / Activo fijo \\
\hline CAF (Composición de activo fijo) & Activo fijo / Activo total \\
\hline LC (Liquidez corriente) & Activo circulante / Pasivo circulante \\
\hline
\end{tabular}

Fuente: elaboración propia

\section{Detalles de la muestra empleada}

La muestra está compuesta de las empresas que cuentan en sus activos con la cuenta activos biológicos. Dichas empresas pertenecen al agronegocio, y actúan en tres segmentos: (1) alimento y bebida; (2) agro-pesca, y (3) papel y celulosa.

Todas las compañías cotizan en la bolsa de Brasil, Bovespa, y son, por tanto, listadas en el sitio Economática, de donde fueron tomados los datos para el cálculo de los indicadores analizados. Un detalle sobre las empresas de la muestra se pude observar en la tabla 2.

TABLA 2

Detalle de las empresas de la muestra

\begin{tabular}{l|l}
\hline Segmentos & Empresas \\
\hline & Souza Cruz, BRF, Josapar, M. Diasbranco, Ambev, JBS, Marfrig, \\
Alimentos y bebidas & Tereos, Minerva, Excelsior, Oderich, Minupar \\
\hline Agro-pesca & Cosan, Brasilagro, SLC Agricola, Sao Martinho \\
\hline Papel y celulosa & Klabin, Fibria, Celul Iarin, Melhor \\
\hline
\end{tabular}

Fuente: elaboración propia

Las empresas pertenecientes al segmento de alimento y bebida se dedican a la comercialización de alimentos y bebidas. Comercializan principalmente los derivados de la proteína animal, así como nutrientes para plantas y animales, y subproductos de origen animal. Algunas de estas empresas también están dedicadas a la refinación de óleos y al procesamiento de carne bovina, porcina, ovina y de aves.

Por su parte, el segmento de agro o producto de la naturaleza tiene como objeto la agricultura y la actividad pecuaria, con un rol importante en la exportación de productos agrícolas de cualquier especie y naturaleza. Algunas de las empresas que lo componen están dedicadas a la actividad agroindustrial, que se vincula con la caña de azúcar, el alcohol y sus derivados. 
En tanto, las empresas de papel y celulosa se dedican a la fabricación, el comercio y la exportación de celulosa, papel y de otros productos oriundos de la transformación de esencias forestales, incluyendo el reciclaje de dichos insumos y productos relacionados con el sector gráfico.

\section{Pasos lógicos y pruebas estadísticas}

En primer lugar, fue necesario clasificar las empresas de acuerdo con sus posibilidades de obtener simultáneamente rentabilidad y liquidez. Para ello se utilizaron los criterios trascritos a continuación, en concordancia con las medias de los indicadores, que fueron calculadas con base en las 231 observaciones. Asimismo, las justificativas asumidas para la formación de grupos de empresas estuvieron basadas en los siguientes criterios:

- Empresas con posibilidad de obtener niveles de rentabilidad y liquidez por encima de los valores medios de las compañías de la muestra en más del $50 \%$ de los años analizados:

$\mathrm{T} 1(\mathrm{RAO} \geq 3,50$ y $\mathrm{LC} \geq 1,45)$

- Empresas cuyos niveles de rentabilidad y liquidez están por debajo de los valores medios en más del $50 \%$ de los años analizados:

$\mathrm{T} 2(\mathrm{RAO}<3,50$ y LC $<1,45)$

Asimismo, fue posible encontrar empresas cuya rentabilidad y liquidez no siguen el mismo comportamiento hasta ahora descrito. Dichas compañías fueron agrupadas en los grupos T3 y T4 según los siguientes criterios:

- Empresas con niveles bajos de rentabilidad, valores por debajo de la media en más del $50 \%$ de los años analizados, y con niveles de liquidez por encima de la media en esos mismos años:

$\mathrm{T} 3(\mathrm{RAO}<3,50$ y $\mathrm{LC} \geq 1,45)$

- Empresas con niveles de rentabilidad por encima de la media en más del $50 \%$ de los años analizados, y con niveles de liquidez por debajo de la media en estos mismos años:

$\mathrm{T} 4(\mathrm{RAO} \geq 3,50$ y $\mathrm{LC}<1,45)$

Se considera que las variables RAF y CAF pueden definir las diferencias que existen entre los dos primeros grupos de empresas T1 y T2. Con el fin de tener la comprobación estadística de la diferencia que hay entre los dos grupos de empresas, se aplicó la prueba Mann-Whitney, habiendo antes contrastado la hipótesis de normalidad de la población por medio de la prueba Kolmogorov-Smirnov. En todos los casos se consideró un nivel nominal de significancia de 0,05 .

\section{Discusión de resultados}

La categoría de empresas agrícolas contiene una amplia diversidad de variables, muchas de ellas exógenas (climatología, cosecha, escala de producción, localización de la producción en tierras más o menos fértiles, entre otras). Dichas variables condicionan particularidades entre las empresas seleccionadas.

Tales particularidades obligan a un ejercicio de abstracción que simplifica, en alguna medida, la realidad objetiva. Dado que se toma consciencia de las limitaciones esenciales que pueden presentar estos grupos para reflejar la complejidad de la realidad del sector en estudio, se presenta una clasificación de los casos seleccionados en la tabla 3. Dicha caracterización corresponde a una tipología de empresas que responde a los requisitos explicados en el anterior apartado. 
TABLA 3

Agrupamiento de las empresas según comportamiento de RAO y LC

\begin{tabular}{l|l|l|l|l|l|l|l|l|l|l|l|l}
\hline Empresas / & & & & & & & & & & & & \\
años & 2005 & 2006 & 2007 & 2008 & 2009 & 2010 & 2011 & 2012 & 2013 & 2014 & 2015 & $\mathrm{~T}$ \\
\hline Souza Cruz & $\mathrm{T} 1$ & $\mathrm{~T} 1$ & $\mathrm{~T} 1$ & $\mathrm{~T} 1$ & $\mathrm{~T} 1$ & $\mathrm{~T} 1$ & $\mathrm{~T} 1$ & $\mathrm{~T} 1$ & $\mathrm{~T} 1$ & $\mathrm{~T} 3$ & $\mathrm{~T} 3$ & $\mathrm{~T} 1$ \\
\hline BRF & $\mathrm{T} 1$ & $\mathrm{~T} 3$ & $\mathrm{~T} 1$ & $\mathrm{~T} 3$ & $\mathrm{~T} 3$ & $\mathrm{~T} 3$ & $\mathrm{~T} 1$ & $\mathrm{~T} 3$ & $\mathrm{~T} 1$ & $\mathrm{~T} 1$ & $\mathrm{~T} 1$ & $\mathrm{~T} 1$ \\
\hline Klabin & $\mathrm{T} 1$ & $\mathrm{~T} 1$ & $\mathrm{~T} 1$ & $\mathrm{~T} 3$ & $\mathrm{~T} 1$ & $\mathrm{~T} 1$ & $\mathrm{~T} 3$ & $\mathrm{~T} 1$ & $\mathrm{~T} 3$ & $\mathrm{~T} 1$ & $\mathrm{~T} 3$ & $\mathrm{~T} 1$ \\
\hline Josapar & $\mathrm{T} 1$ & $\mathrm{~T} 1$ & $\mathrm{~T} 1$ & $\mathrm{~T} 1$ & $\mathrm{~T} 3$ & $\mathrm{~T} 3$ & $\mathrm{~T} 3$ & $\mathrm{~T} 1$ & $\mathrm{~T} 3$ & $\mathrm{~T} 3$ & $\mathrm{~T} 3$ & $\mathrm{~T} 3$ \\
\hline Fibria & $\mathrm{T} 1$ & $\mathrm{~T} 1$ & $\mathrm{~T} 1$ & $\mathrm{~T} 1$ & $\mathrm{~T} 1$ & $\mathrm{~T} 1$ & $\mathrm{~T} 3$ & $\mathrm{~T} 3$ & $\mathrm{~T} 2$ & $\mathrm{~T} 3$ & $\mathrm{~T} 3$ & $\mathrm{~T} 1$ \\
\hline M. Diasbranco & $\mathrm{T} 1$ & $\mathrm{~T} 1$ & $\mathrm{~T} 1$ & $\mathrm{~T} 4$ & $\mathrm{~T} 1$ & $\mathrm{~T} 1$ & $\mathrm{~T} 1$ & $\mathrm{~T} 1$ & $\mathrm{~T} 1$ & $\mathrm{~T} 1$ & $\mathrm{~T} 1$ & $\mathrm{~T} 1$ \\
\hline Ambev & $\mathrm{T} 1$ & $\mathrm{~T} 1$ & $\mathrm{~T} 4$ & $\mathrm{~T} 4$ & $\mathrm{~T} 1$ & $\mathrm{~T} 4$ & $\mathrm{~T} 1$ & $\mathrm{~T} 1$ & $\mathrm{~T} 1$ & $\mathrm{~T} 4$ & $\mathrm{~T} 3$ & $\mathrm{~T} 1$ \\
\hline JBS & $\mathrm{T} 1$ & $\mathrm{~T} 1$ & $\mathrm{~T} 1$ & $\mathrm{~T} 1$ & $\mathrm{~T} 1$ & $\mathrm{~T} 1$ & $\mathrm{~T} 3$ & $\mathrm{~T} 3$ & $\mathrm{~T} 3$ & $\mathrm{~T} 3$ & $\mathrm{~T} 3$ & $\mathrm{~T} 1$ \\
\hline Marfig & $\mathrm{T} 3$ & $\mathrm{~T} 3$ & $\mathrm{~T} 3$ & $\mathrm{~T} 3$ & $\mathrm{~T} 1$ & $\mathrm{~T} 2$ & $\mathrm{~T} 2$ & $\mathrm{~T} 3$ & $\mathrm{~T} 3$ & $\mathrm{~T} 3$ & $\mathrm{~T} 2$ & $\mathrm{~T} 3$ \\
\hline Tereos & $\mathrm{T} 2$ & $\mathrm{~T} 2$ & $\mathrm{~T} 2$ & $\mathrm{~T} 2$ & $\mathrm{~T} 2$ & $\mathrm{~T} 2$ & $\mathrm{~T} 2$ & $\mathrm{~T} 2$ & $\mathrm{~T} 2$ & $\mathrm{~T} 2$ & $\mathrm{~T} 2$ & $\mathrm{~T} 2$ \\
\hline Minerva & $\mathrm{T} 2$ & $\mathrm{~T} 1$ & $\mathrm{~T} 1$ & $\mathrm{~T} 3$ & $\mathrm{~T} 1$ & $\mathrm{~T} 3$ & $\mathrm{~T} 3$ & $\mathrm{~T} 3$ & $\mathrm{~T} 3$ & $\mathrm{~T} 3$ & $\mathrm{~T} 3$ & $\mathrm{~T} 3$ \\
\hline Cosan & $\mathrm{T} 3$ & $\mathrm{~T} 3$ & $\mathrm{~T} 3$ & $\mathrm{~T} 3$ & $\mathrm{~T} 3$ & $\mathrm{~T} 1$ & $\mathrm{~T} 1$ & $\mathrm{~T} 3$ & $\mathrm{~T} 2$ & $\mathrm{~T} 1$ & $\mathrm{~T} 1$ & $\mathrm{~T} 3$ \\
\hline Excelsior & $\mathrm{T} 1$ & $\mathrm{~T} 1$ & $\mathrm{~T} 1$ & $\mathrm{~T} 1$ & $\mathrm{~T} 1$ & $\mathrm{~T} 3$ & $\mathrm{~T} 4$ & $\mathrm{~T} 4$ & $\mathrm{~T} 1$ & $\mathrm{~T} 1$ & $\mathrm{~T} 2$ & $\mathrm{~T} 1$ \\
\hline Celul larin & $\mathrm{T} 2$ & $\mathrm{~T} 2$ & $\mathrm{~T} 2$ & $\mathrm{~T} 4$ & $\mathrm{~T} 4$ & $\mathrm{~T} 1$ & $\mathrm{~T} 2$ & $\mathrm{~T} 1$ & $\mathrm{~T} 1$ & $\mathrm{~T} 2$ & $\mathrm{~T} 2$ & $\mathrm{~T} 2$ \\
\hline Brasilagro & $\mathrm{T} 3$ & $\mathrm{~T} 3$ & $\mathrm{~T} 3$ & $\mathrm{~T} 3$ & $\mathrm{~T} 4$ & $\mathrm{~T} 1$ & $\mathrm{~T} 1$ & $\mathrm{~T} 1$ & $\mathrm{~T} 2$ & $\mathrm{~T} 1$ & $\mathrm{~T} 1$ & $\mathrm{~T} 1$ \\
\hline Minupar & $\mathrm{T} 3$ & $\mathrm{~T} 1$ & $\mathrm{~T} 1$ & $\mathrm{~T} 3$ & $\mathrm{~T} 3$ & $\mathrm{~T} 3$ & $\mathrm{~T} 1$ & $\mathrm{~T} 2$ & $\mathrm{~T} 2$ & $\mathrm{~T} 2$ & $\mathrm{~T} 2$ & $\mathrm{~T} 2$ \\
\hline Sacich & $\mathrm{T} 2$ & $\mathrm{~T} 3$ & $\mathrm{~T} 2$ & $\mathrm{~T} 3$ & $\mathrm{~T} 3$ & $\mathrm{~T} 2$ & $\mathrm{~T} 3$ & $\mathrm{~T} 1$ & $\mathrm{~T} 2$ & $\mathrm{~T} 2$ & $\mathrm{~T} 2$ \\
\hline
\end{tabular}

Fuente: elaboración propia

En la anterior tabla se puede observar que el $45 \%$ de las empresas tienen una tendencia favorable en lo que atañe a la obtención simultánea de rentabilidad y liquidez durante el período en estudio. En contraste, el 30\% coinciden en mostrar resultados desfavorables para ambos indicadores. Por último, en un grupo de empresas que corresponde al $25 \%$ del total analizado, se favorece la liquidez mientras se perjudica la rentabilidad.

A lo largo de los dos últimos años se observa una sensible disminución de la cantidad de empresas del grupo 1 , y un discreto traslado de estas a los grupos 2 y 3: un fenómeno en el que el grupo 2 se impone. Resulta destacable que, de forma general, los años 2008, 2014 y 2015 marquen la diferencia en la ocurrencia de este evento.

En la tabla 4 se puede observar una demostración estadística de la correlación que comparten estos indicadores y los factores que los componen. 
TABLA 4

Resultados de coeficientes de correlación entre las variables de estudio

\begin{tabular}{|c|c|c|c|c|c|c|}
\hline & & MO & RA & RAO & LC & RAF \\
\hline \multirow[t]{4}{*}{ MO } & Correlación de Spearman & 1 & $-0,507^{*}$ & $0,631^{*}$ & 0,205 & $0,559^{*}$ \\
\hline & Nivel de significancia (2- & & 0,000 & 0,000 & 0,078 & 0,001 \\
\hline & tailed) & - & & & & \\
\hline & Total de observaciones & 231 & 231 & 231 & 231 & 231 \\
\hline \multirow[t]{3}{*}{ RA } & Correlación de Spearman & $-0,507^{\prime \prime}$ & 1 & 0,256 & $0,435^{\prime \prime}$ & $0,235^{\circ}$ \\
\hline & $\begin{array}{l}\text { Nivel de significancia (2- } \\
\text { tailed) }\end{array}$ & 0,000 & - & 0,001 & 0,041 & 0,041 \\
\hline & Total de observaciones & 231 & 231 & 231 & 231 & 231 \\
\hline \multirow[t]{3}{*}{ RAO } & Correlación de Spearman & $0,631^{\prime \prime}$ & 0,256 & 1 & $0,590^{27}$ & $0,671^{n+}$ \\
\hline & $\begin{array}{l}\text { Nivel de significancia (2- } \\
\text { tailed) }\end{array}$ & 0,000 & 0,011 & - & 0,010 & 0,000 \\
\hline & Total de observaciones & 231 & 231 & 231 & 231 & 231 \\
\hline \multirow[t]{3}{*}{ LC } & Correlación de Spearman & 0,205 & $0,435^{\prime \prime}$ & $0,590^{\prime \prime}$ & 1 & $0,435^{\circ}$ \\
\hline & $\begin{array}{l}\text { Nivel de significancia (2- } \\
\text { tailed) }\end{array}$ & 0,078 & 0,041 & 0,010 & - & 0,041 \\
\hline & Total de observaciones & 231 & 231 & 231 & 231 & 231 \\
\hline \multirow[t]{3}{*}{ RAF } & Correlación de Spearman & $0,559^{* *}$ & $0,235^{\circ}$ & $0,671^{*}$ & $0,435^{\circ}$ & 1 \\
\hline & $\begin{array}{l}\text { Nivel de significancia (2- } \\
\text { tailed) }\end{array}$ & 0,001 & 0,041 & 0,000 & 0,041 & - \\
\hline & Total de observaciones & 231 & 231 & 231 & 231 & 231 \\
\hline
\end{tabular}

Los resultados expuestos en la anterior tabla reflejan una correlación positiva y estadísticamente significativa entre liquidez y rentabilidad (0,590). Dichas conclusiones coinciden con las obtenidas en otros estudios (Correia, 2003; Melgarejo y Vera, 2010; Pimentel \& Lima, 2011). Por su parte, los resultados de ROA están más influenciados por los del margen $(0,631)$ que por los de RA $(0,256)$. Esto evidencia, una vez más, la existencia de trade-off entre MO y RA $(-0,507)$, lo cual confirma los hallazgos de diferentes autores que han documentado que el margen tiene mayor influencia sobre la rentabilidad (Díaz \& Morillas, 2004; Monterrey \& Sánchez, 2011).

Muchos de los estudios teóricos y prácticos que se dedican al vínculo existente entre la rentabilidad y la liquidez, han revelado una relación divergente; sin embargo, el presente estudio revela una relación positiva. Con el fin de evaluar las posibles condiciones de esta convergencia, se incluyó la variable RAF en el análisis de la correlación. Lo anterior permitió observar que los niveles de correlación de esta con el resto de las variables son positivos y estadísticamente significativos. En suma, esta variable junto con la denominada CAF pueden llevar a diferenciar a los dos grupos de empresas en las que concuerdan los comportamientos de la rentabilidad y la liquidez.

El siguiente análisis tiene el propósito de indagar si los grupos de empresas pueden diferenciarse. Para ello, en primer lugar se aplicó la prueba Kolmogorov-Smirnov, la cual ofreció los resultados que se muestran en la tabla 5. 
TABLA 5

\begin{tabular}{l|l|l|l}
\multicolumn{3}{c}{ Resultado de la prueba Kolmogorov-Smirnov } \\
\hline \multirow{2}{*}{ Grupos } & Variables & promedio & p-valor \\
\hline & CAF & 38,2 & \\
$\mathbf{1}$ (T2) & RAF & 1,48 & \multirow{2}{*}{0,00} \\
\hline & CAF & 56,2 & \\
$\mathbf{2}$ (T1) & RAF & 2,09 & \\
\hline
\end{tabular}

Fuente: elaboración propia

Total de observaciones: 165

Los resultados ofrecidos en la tabla 5 favorecen la independencia de las muestras. De acuerdo con el nivel de significancia obtenido, puede decirse que los datos no siguen una distribución normal. A continuación se aplica la prueba Mann-Whitney, cuyos resultados se pueden observar en la tabla 6.

TABLA 6

Resultados de la prueba Mann-Whitney

\begin{tabular}{l|l|l|l}
\hline Variables & Grupos & Mediana & p-valor \\
\hline CAF & 1 & 33,7 & 0,02 \\
\hline RAF & 2 & 1,05 & \\
\hline CAF & 1 & 48,5 & 0,04 \\
\cline { 1 - 2 } RAF & 2 & 1,88 & \\
\hline
\end{tabular}

Fuente: elaboración propia

Total de observaciones: 165

En general, es posible argumentar que las empresas que componen el grupo 1 (empresas clasificadas como T1) son diferentes de las que componen el grupo 2 (empresas clasificadas como T2). Los resultados expuestos en la tabla 6 permiten observar que los rangos promedios de RAF y de CAF son mayores que los de las empresas del grupo 1: en ese sentido, hacia ello mismo se inclinan las medianas de ambos grupos. De acuerdo con dichos resultados es plausible sugerir que, al monitorear el comportamiento de estas variables, sería posible distinguir y direccionar una relación positiva entre los indicadores de la rentabilidad y la liquidez. 


\section{Conclusiones}

1. Se pone de manifiesto que rentabilidad y liquidez no son variables necesariamente relacionadas negativamente, como señala parte de la revisión bibliográfica.

2. Según los criterios utilizados, en el $75 \%$ de los casos analizados RAO (rentabilidad) y LC (liquidez) reflejan dependencia directas con relación a las medias. En el $45 \%$ de los casos estudiados los resultados de ambos indicadores son mejores que la media de la muestra, y el $30 \%$ manifiesta los peores resultados de forma conjunta en los dos indicadores analizados con relación a la media. Solo en un $25 \%$ de los casos analizados las relaciones que hay entre rentabilidad y liquidez fueron inversas con relación a las medias.

3. En el sector del agronegocio se encuentran evidencias de la existencia óptima de rentabilidad y liquidez en empresas que venden más por cada unidad monetaria en activos fijos, y que presentan mayor proporción de este tipo de activo en relación con el circulante.

4. Es necesario considerar las limitaciones al alcance de las pruebas realizadas, dada la heterogeneidad del universo de empresas pertenecientes al agronegocio y la ineludible influencia de variables exógenas (temporalidad, cosecha, climatología). Al interior del sector existen notables diferencias en cuanto atañe al tipo de actividad y producto, nivel de especialización, superficie terrestre en explotación (no siempre con la misma productividad del trabajo) y el tipo de mercado en el que opera la empresa.

5. Estudios posteriores pueden conseguir mayor nivel de profundidad estableciendo niveles óptimos de activos, de acuerdo con las particularidades de los grupos de empresas (empresa productora o comercializadora). En ese contexto, la delimitación por períodos permitirá análisis temporales más profundos y que cuenten con mayor nivel de particularización, mediante la identificación de la influencia de años de malas cosechas, así como según otros elementos propios de las fases productivas que versen sobre la estructura de capital, el nivel de endeudamiento y otros aspectos.

\section{Agradecimientos}

El estudio fue amparado por el proyecto Análise das demonstrações contábeis como subsídio ao processo decisório, código: 0036/2017, de la entidad Universidade Federal de Uberlândia.

\section{Referencias}

Acosta, M., Correa, A. y González, A.(2002). Factores determinantes de la rentabilidad financiera de las pymes. Revista Española de Financiación y Contabilidad, XXXI(112), 395-430.

Afza, T. \& Nazir, M. (2007). Is it better to be aggressive or conservative in managing working capital? Journal of Quality and Technology Management, 3(2), 11-21.

Aguilar, I., Ruíz, M., \& Santana, D. J. (2000). Análisis de los factores determinantes de la rentabilidad. Una propuesta metodológica. Actualidad Financiera, 6(4), 3-15.

Amat, O. (2001). Análisis de estados financieros. Fundamentos y aplicaciones. España: Gestión.

Anake, F., Ikes, J., \& Menyo, S. (2015). Determinantes del capital de trabajo. International Journal of Economics, Commerce and Management, III(2), 1-11.

Barry, P., Ellinger, P., \& Hopkin, J. (1995). Financial Management in Agriculture. USA: Sixth.

Braga, R., Nossa, V. \& Marques, L. (2004). Uma proposta para a analise integrada da liquidez. Revista Contabilidade \& Finanças, Edição Especial, 51-64. 
Brito, E. (2010). Um estudo sobre a subjetividade na mensuração do valor justo na atividade da pecuária bovina (Dissertação de Mestrado). Universidade São Pablo, São Pablo, Brasil.

Cáceres, D. (2015). Tecnología agropecuaria y agronegocios. La lógica subyacente del modelo tecnológico dominante. Mundo Agrario, 16(31). Recuperado de http://www.mundoagrario.unlp.edu.ar/article/view/MAv16n31a08

Caligaris, G. (2015). Concentración y centralización del capital agrario en la región pampeana. El caso de los grandes pooles de siembra. Mundo Agrario, 16(31), 1-34.

Chordia, T., Roll, R., \& Subrahmanyam, A. (2000). Market liquidity and trading activity. Journal of Finance, 56(2), 501-530.

Correia, T. P. (2003). Determinantes da Estrutura de Capital das Empresas Familiares Portuguesas (Dissertação de Mestrado em Finanças Empresariais). Universidade do Algarve, Portugal.

Cuervo, A. \& Rivero, P. (1999). El análisis económico-financiero de la empresa. Revista española de financiación y contabilidad, 16(49), 15-33.

Deloof, M. (2003). Does working capital management affect profitability of Belgian firms? Journal of Business Finance \& Accounting, 30(3-4), 573-588.

Dong, H. P. y Su, J. T. (2010). The relationship between working capital management and profitability: A Vietnam case. International Research Journal of Finance and Economics, 49, 62-71.

Eljelly, A. (2004). Liquidity - Profitability Trade Off: An empirical investigation in an emerging market. International Journal of Commerce \& Management, 14(2), 48-61.

Fernández, F., Ferreira, M. y Rodríguez, E. (2014). Análisis de rentabilidad utilizando el modelo DUPONT. Revista de Gestão em Sistemas de Saúde - RGSS, 3(2), 21-37.

Filbeck, G. \& Krueger, T. (2007). “Working Capital Survey”: Do select firms word for shareholders? Quarterly Journal of Business and Economics, 46(2), 3-22.

García., T. \& Martínez, P. (2007). Effects of working capital management on SME profitability. International Journal of Managerial Finance, 3(2), 164-177.

Gill, A., Biger, N. y Mathur, N. (2010). The Relationship between Working Capital Management and Profitability: Evidence from the United States. Business and Economics Journal, 2, 1-9.

Hall, R., Beck, F., \& Toledo, J. (2012). Do impacto da crise subprime nas empresas do agronegócio brasileiro listadas na BM\&FBovespa. Custos e @gronegócio on line, 9(1), 3-16.

Hasbrouck, J. \& Seppi, D. J. (2001). Common factors in prices, order flows, and liquidity. Journal of Financial Economics, 59(3), 383- 411.

Hirshleifer, D., How, K., Teoh, S., \& Zhang, W. (2004). Do investor overvalue firms with bloated balance sheets? Journal of Accounting and Economics, 38, 297-331.

Howorth, C. \& Westhead, P. (2003). The focus of working capital management in UK small firms. Management Accounting Research, 14, 94-111.

Hussain, A., Farooz., S. U., \& Khan, K. U. (2012). Aggressiveness and Conservativeness of Working Capital: A case of Pakistan Manufacturing Sector. European Journal of Scientific Research, 73(2), 171-182.

Ikram, H., Mohammad, S., Khalid, Z., \& Zaheer, A. (2011). The relationship between Working Capital Management and Profitability: A case study of Cement Industry in Pakistan. Mediterranean Journal of Social Sciences, 2(2), 63-74.

Iudícibus, S. (1998). Análises de Balanço. São Paulo: Atlas.

Jarvis, R., Kitching, J., Curran, J., \& Lightfoot. (1996). The financial management of small firms: An alternative perspective. ACCA Research Report, 49, 45-67.

Jiménez, F., \& Lozano, J. (2014). Algunas consideraciones sobre la relación entre orientación al mercado y rentabilidad. Revista Investigación y Marketing, 64, 34-39.

Koshio, S. \& Nakamura, W. (2013). Efeitos de riscos de mercado e regime de caixa sobre a relação liquidezrentabilidade. Práticas em Contabilidade e Gestão, 1(1), 40-65. 
Lazaridis, I. y Trifonidis, D. (2006). Relationship between working capital management and profitability of listed companies in the Athens Stock Exchange. Journal of Financial Management and Analysis, 19(1), 26-35.

Marques, J. A. y Braga, R. (1995). Análise Dinâmica do Capital de Giro, o Modelo Fleuriet. RAE - Revista de Administração de Empresas, 35(3), 49-63.

Martins, E., Miranda, G. J., \& Alvez, J. (2014). Análise das Demonstrações Contábeis. São Paulo: Atlas.

Matarazzo, D. C. (2008). Análise financeira de balanços: abordagem básica e gerencial. São Paulo: Atlas.

Mathuva, D. (2009). The influence of working capital management components on corporate profitability: A survey of Kenyan listed firms. Research Journal of Business Management, 4(1), 1-11.

Medeiros, O. R. \& Rodrigues, F. (2004). Questionando empiricamente a validade do Modelo Fleuriet. Revista de Administração e Contabilidade da Unisinos, 1(2), 25-32.

Mehtab, M. \& Marsap, A. (2015). Factores determinantes de las necesidades de capital de trabajo. International Journal of Economics and Finance, 7(2), 280-292.

Melgarejo,Z. y Vera, M. (2010). Análisis de la relación entre rentabilidad y riesgo de las sociedades laborales y sociedades mercantiles convencionales. Revista de la Facultad de Ciencias Económicas y Administrativas, XI(1), 97-116.

Mohammadi, M. (2009). The impact of working capital management on corporate profitability. Management Journal, $1(14), 17-38$.

Mona, A. M. (2012). The impact of working capital management policies on firm's profitability and value: The case of Jordan. Journal of Finance and Economics, 85, 147-153.

Monterrey, J. \& Sánchez, A. (2011). Persistencia de la rentabilidad. Un estudio de sus factores determinantes. Revista española de financiación y contabilidad, XI(150), 287-317.

Muhammad, M. y Akin, M. (2015). Determinant factors and working capital requirement. International Journal of Economics and Finance, 7(22), 280-292.

Padachi, K. (2006). Trends in working capital management and its impact on firms' performance: An analysis of Mauritian small manufacturing firms. International Review of Business Research, 2(2), 45-58.

Padoveze, C. L. \& Benedicto, G. C. (2007). Análise das Demonstrações Financeiras (2a Ed.). São Paulo: Thomson.

Pérez, J. A., Castro, O. y García , B. (2009). Gestión del capital circulante y rentabilidad en pymes. Revista de Contabilidady Dirección, 9, 119-140.

Pimentel, R., Braga, R. \& Pereira, S. (2005). Interação entre rentabilidade e liquidez: um estudo exploratório. Revista de Contabilidade do Mestrado em Ciências Contábeis da UERJ, 10(2), 83-96.

Pimentel, R. y Lima, I. (2011). Relação trimestral de longo prazo entre os indicadores de liquidez y rentabilidade: evidencias de empresas do setor têxtil. Revista Adm. São Paulo, 46(3), 275-289.

Pimentel, R. ( 6 al 8 de septiembre de 2008). Dilema entre Liquidez e Rentabilidade: um Estudo Empírico em Empresas Brasileiras. En XXXII Encontro de ANPAD. Encuentro llevado a cabo en Rio de Janeiro, Brasil.

Porlles, J. (2015). Flujo de fondos: poderosa herramienta de gestión. Gestión en el Tercer Milenio. Revista de Investigación de la Facultad. de Ciencias Administrativas, UNMSM, 8(16), 13-28.

Rahneman, A. \& Nasir, M. (2007). Working capital management and profitability. Case of Pakistani Firms. International Review of Business Research Papers, 3, 79-100.

Samiloglu, F. y Demirgünes, K. (2008). The effect of working capital management on firm profitability: Evidence from Turkey. International Journal of Applied Economics and Finance, 2(1), 44-50.

Sánchez, J. \& Bernabe, P. (2002). La rentabilidad de la empresa española: un estudio sobre la década de los noventa. Partida doble, (131), 98-111.

Shin, H. y Soenen, L.(1998). Efficiency of working capital management and corporate profitability. Financial Practice and Education, 8(2), 37-45.

Souza, S. (2008). Risco de crédito, capital de giro e solvência empresarial: um estudo na indústria brasileira de transformação de cobre. Universo Contábil, Blumenau, 4(3), 30-35.

Symons, R. (2005). Accounting for farms. London: Tottel Publishing. 
Talha, M., Christopher, S., \& Kamalavalli, A. (2010). Sensitivity of profitability to working capital management: A study of Indian corporate hospitals. International Journal of Managerial and Financial Accounting, 2(3), 213-227.

Van Horne, J. \& Wachowicz, J. (2014). Fundamental of financial management. New York: Prentice Hall Publishers.

Vercelli, A. (2000). Preferência pela liquidez e valor de opção. Revista Economia e Sociedade, 12, 21-40.

Vértiz, P. (2015). El avance de los agronegocios en regiones marginales del agro pampeano: concentración de la producción y tensiones entre las fracciones del capital agrario. Mundo Agrario, 16(33), 1-33.

\section{Notas}

* Artículo de investigación científica y tecnológica

\section{Licencia Creative Commons CC BY 4.0}

Para citar este artículo: Vázquez Carrazana, X. y Rech, I. J., Miranda, G. J. y Tavares, M. (2017). Convergencias entre la rentabilidad y la liquidez en el sector del agronegocio. Cuadernos de Contabilidad, 18(45), 152-165. https://doi.org/10.11144/Javeriana.cc18-45.crls 